\title{
THE VALUE OF 3D PRINTING IN ORTHOPAEDICS
}

\section{The Value of 3D Printing in Orthopaedics}

Umesh Persad $^{\mathbf{1}}$, Marlon Mencia ${ }^{2}$

1-Mechanical Engineering, Manufacturing, and Entrepreneurship Unit, The University of Trinidad and Tobago, Brechin Castle, Couva, Trinidad and Tobago, West Indies.

${ }^{2}$ Department of Clinical Surgical Sciences, School of Medicine, The University of The West Indies, St. Augustine, Trinidad and Tobago, West Indies.

\section{Corresponding Author:}

Dr Umesh Persad

Mechanical Engineering, Manufacturing, and

Entrepreneurship Unit,

The University of Trinidad and Tobago,

Brechin Castle, Couva,

Trinidad and Tobago,

West Indies

Email: umesh.persad@utt.edu.tt

DOI: $\underline{10.48107 / C M J .2020 .12 .005}$

Copyright:

(c) (i)

This is an open access article under the terms of the Creative Commons Attribution License which permits use, distribution and reproduction in any medium, provided the original work is properly cited.
The use of three-dimensional (3D) printing technology, technically known as additive manufacturing, is rapidly growing in the field of Orthopaedics. ${ }^{1}$ Three-dimensional printing enables the manufacture of 3D objects with complex geometries by depositing a material layer by layer until a full physical model is developed. The most common types of 3D printing in use are Fused Deposition Modelling (FDM) and Stereolithography (SLA). FDM works by heating and extruding a polymer onto a print bed, layer by layer to build the 3D model. SLA works by using a laser to cure a photosensitive liquid resin layer by layer within a vat of the resin. ${ }^{1}$ The input to a 3D printer is a Standard Triangle Language (STL) file that describes the surface geometry in terms of triangulated surfaces. The STL files could be generated from external 3D anatomical scanners, computerized tomography (CT) or magnetic resonance imaging (MRI) scans and with the use of a computer-aided design (CAD) software program as used in Engineering. ${ }^{2}$

Three-dimensional printing is available either via a commercial manufacturing service or the investment in a 3D print lab directly situated in a clinic or hospital. ${ }^{3}$ For all medical applications, there is usually a scan-design-print pipeline that involves first acquiring patient 3D data, processing that $3 \mathrm{D}$ data to isolate the features of interest and then finally printing the $3 \mathrm{D}$ data via a $3 \mathrm{D}$ printer. ${ }^{2,3}$ In the drive towards individualised orthopaedics, the following have been identified as key areas for the application of 3D printing: (1) anatomical models, (2) prosthetics and orthotics (3) custom implants and instruments and (4) living tissues. ${ }^{3}$ We discuss the relevance of these areas to current orthopaedic practices in Trinidad and Tobago, as well as future applications.

The use of 3D printed anatomical models is becoming more commonplace in orthopaedic surgical practice especially when there is the need to visualise complex deformities and fractures. The models can be used for preoperative planning, pre-fitting of implants, collaborative surgical team communication and patient communication and consultation., ${ }^{4,5}$ The use of 3D printed anatomical models has been shown to be 
effective in understanding the complex spatial relationships required for effective surgery, resulting in shorter surgical times with savings in overall cost. ${ }^{6-8}$ This application is currently available in Trinidad and Tobago through manufacturers and Fab Labs. With desktop 3D printers becoming more affordable, this area is ripe for wide scale adoption.

Customised prosthetics and orthotics are another area in which 3D printing is proving to be useful. ${ }^{9,10}$ The key advantage of 3D printing in this instance is the ability to customise and manufacture a prosthetic or orthotic device at a reasonable cost. This ability to personalise the colour, shape and size of the device is useful especially for children, where customisation could lead to increased compliance. ${ }^{10}$ In addition, as a child grows, there is a need for device adjustment or a new device, and 3D printed designs could easily be digitally modified to account for this. There are many open-source designs available that increase the accessibility of prosthetic devices in resource-constrained environments. It therefore becomes more economically feasible to 3D print prosthetic devices and support them in local environments rather that sourcing them externally.

Figure I shows examples of locally manufactured 3D printed anatomical models and upper limb prosthetics.
The anatomical models have proven to be effective in surgical planning and patient education. The models were produced using open source imaging processing software and a Fused Deposition Modelling (FDM) 3D printer. The model itself was made of the bioplastic Polylactic Acid (PLA) which differs from other thermoplastic polymers in that it is made from renewable resources like corn starch or sugar cane. The advantage of PLA as a bioplastic in a developing country context is that it naturally degrades when exposed to the environment, proving to be a more sustainable and environmentally-friendly material.

The hand prosthetics in Figure I were made using the popular open source e-NABLE Raptor Reloaded prosthetic hand design 3D printed in PLA. It was found that the prosthetic hands provided basic functionality, but the design required adjustments to account for assembly issues and high activation forces for the user. The smaller of the two prosthetic hands was made for a five-year-old child who lost his right hand in a scratch bomb accident. Although it was initially well received by the patient, the hand was only used intermittently over the long term primarily because of its limited functionality. This points to an opportunity for the design improvement of existing open source prosthetic hands and the development of new 3D printable prosthetic hands that could be designed, made and supported locally.

Figure 1. Locally manufactured 3D printed models and prosthetics

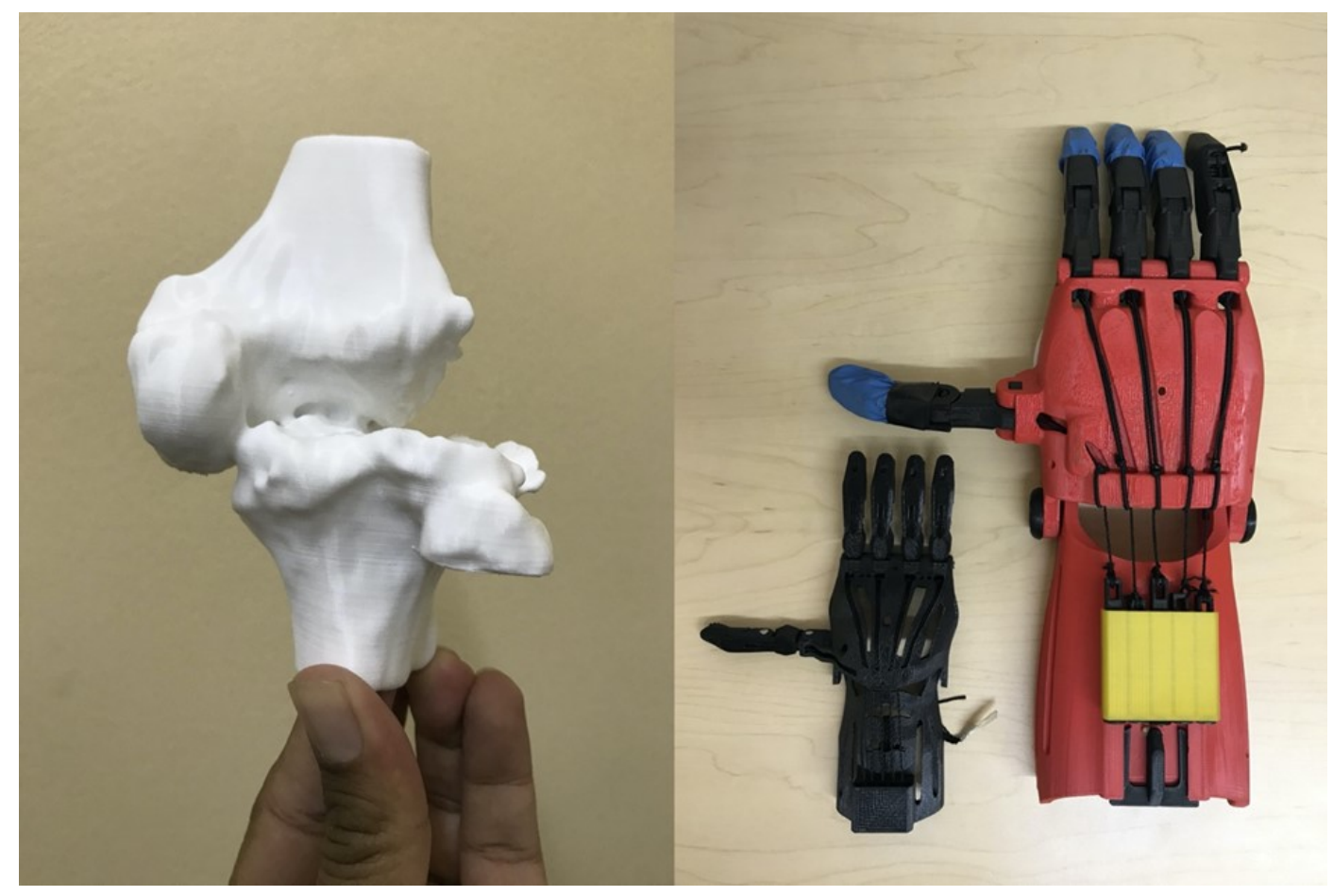


Personalized instruments and implants that map directly on to the patient's anatomy represent an exciting application of the 3D printing process. ${ }^{3,4}$ Improved operating theatre efficiency, minimally invasive surgical approaches and improved clinical outcomes are some of the proposed advantages of this new technology. Most international major implant manufacturers offer the option of customised implants with a 3 to 4 week production time that could be accessed locally with the aid of a concierge service. Currently, there are no 3D metal printers in Trinidad and Tobago, and the requirements of quality, sterilisation and a regulated industry may hinder development locally in this area for some time.

The manufacture of living tissues also known as 3D bioprinting refers to and has quickly become one of the most innovative segments of the 3D printing industry. The ability to combine various cell types, growth factors and structural material in a specific anatomical shape to create a biological tissue has vast potential. With osteoarthritis affecting $19 \%$ of Americans > 45 years of age and prevalence rates rising, the applicability to $3 \mathrm{D}$ bio-printing of cartilage and bone represents an area of intense current research. Regenerative medicine as it relates to orthopaedics is in the early developmental phase in the United States, making it unlikely that these techniques will be available locally in the foreseeable future.

In summary, 3D printing technology is poised to transform the current practice of orthopaedic surgery worldwide. With the work initiated by the authors, some applications are already being adopted locally in the areas of patient-specific anatomical models and prosthetics/ orthotics. The overall demand for 3D printed medical products locally and regionally is currently not known, as technology adoption of medical digital design and 3D printing is in its infancy locally. However, initial feedback indicates that there will be a role for this technology to play given the benefits such as reduced surgical times and cost. Additionally, patients requiring prosthetics or long-term orthotics can also benefit from a personalised approach.

Expansion of local 3D printing services for orthopaedic applications requires close collaboration between surgeons, radiologists and engineers. ${ }^{4}$ There are only a handful of local companies that can provide reliable 3D printing services at this time. However, they lack the expertise to convert medical imaging data to 3D models for 3D printing and to design products on scanned surface anatomy for orthotic/prosthetic production. 3D printing technology is also evolving rapidly, with new techniques of 3D printing, lower cost machines, and new printing materials becoming available. Local manufacturing companies are looking at the market to decide if investing in 3D printing technologies could serve a range of markets. If these services are increasingly utilised by medical professionals, then it is expected that they will become rooted in orthopaedic practice as is occurring in other areas such as dentistry.

At this time, it is recommended that radiologists and surgeons initially collaborate with academic institutions and established manufacturing companies with engineering expertise for service reliability. Eventually, as experience with the technology develops, the possibility of establishing 3D print labs within hospital radiology departments and surgical practices could be considered. Currently, there needs to be education and training for both engineers and medical professionals on the scan to print pipeline and the use of available software tools. The value of 3D printing technology to surgeons and patients would outweigh the initial set-up costs of equipment purchase and ongoing material supplies once there is increased demand. Although available in developed countries for some time, it is only now possible through collaborative efforts that smaller developing countries can utilise 3D printing for orthopaedic applications with local design and manufacturing.

Ethical Approval Statement: not applicable to viewpoint article.

Informed Consent: not applicable to viewpoint article.

Conflict of Interest: authors have no conflict of interest to declare.

Funding Statement: not applicable to viewpoint article.

Author Contribution Statement: both authors contributed equally to developing and writing the manuscript. 


\section{References}

1. Skelley NW, Smith MJ, Ma R, Cook JL. Threedimensional Printing Technology in Orthopaedics. JAAOS-Journal of the American Academy of Orthopaedic Surgeons. 2019;27(24):918-25.

2. Marro A, Bandukwala T, Mak W. Three-dimensional printing and medical imaging: a review of the methods and applications. Current problems in diagnostic radiology. 2016;45(1):2-9.

3. Wong KC. 3D-printed patient-specific applications in orthopedics. Orthopedic research and reviews.

2016;8:57.

4. Matsumoto JS, Morris JM, Foley TA, Williamson EE, Leng S, McGee KP, Kuhlmann JL, Nesberg LE, Vrtiska TJ. Three-dimensional physical modeling: applications and experience at Mayo Clinic. Radiographics.

2015;35(7):1989-2006.

5. Chepelev L, Wake N, Ryan J, Althobaity W, Gupta A, Arribas E, Santiago L, Ballard DH, Wang KC, Weadock W, Ionita CN. Radiological Society of North America (RSNA) 3D printing Special Interest Group (SIG): guidelines for medical 3D printing and appropriateness for clinical scenarios. 3D printing in medicine. 2018;4(1):1-38.

6. Chen C, Cai L, Zheng W, Wang J, Guo X, Chen H. The efficacy of using 3D printing models in the treatment of fractures: a randomised clinical trial. $B M C$ musculoskeletal disorders. 2019;20(1):65.

7. Ballard DH, Mills P, Duszak Jr R, Weisman JA, Rybicki FJ, Woodard PK. Medical 3D Printing Cost-Savings in Orthopedic and Maxillofacial Surgery: Cost Analysis of Operating Room Time Saved with 3D Printed Anatomic Models and Surgical Guides. Academic radiology. 2019.

8. Huang Z, Song W, Zhang Y, Zhang Q, Zhou D, Zhou $X$, He $Y$. Three-dimensional printing model improves morphological understanding in acetabular fracture learning: A multicenter, randomized, controlled study. PloS one. 2018;13(1).

9. Ten Kate J, Smit G, Breedveld P. 3D-printed upper limb prostheses: a review. Disability and

Rehabilitation: Assistive Technology. 2017;12(3):300-

14.
10. Manero A, Smith P, Sparkman J, Dombrowski M, Courbin D, Kester A, Womack I, Chi A.

Implementation of 3D printing technology in the field of prosthetics: Past, present, and future. International journal of environmental research and public health. 2019;16(9): 1641. 